\title{
Spacetime Curvature is Important for Cosmology Constrained with Supernova Emissions
}

\author{
Ahmet M. Öztaş • Michael L. Smith • Jan Paul
}

Received: 6 October 2007 / Accepted: 30 January 2008 / Published online: 14 February 2008

(C) Springer Science+Business Media, LLC 2008

\begin{abstract}
We investigate universe expansion models as functions of emission frequency ratio decline rather than redshift $z$, using the latest on-line, self-consistent data from 192 supernovae. We present results for simpler and some current models of cosmology, including those with dark energy (standard model) and a recent model correcting for the effect of a small time-dependent, emission frequency increase with lookback. This new model, with a gentle lookback decline of the Planck constant, and the standard model fit the data with similar confidence according to Bayesian Information Criteria. The standard model tends towards solutions high in matter density while remaining flat, but models without dark energy tend towards dilute universes with significant spacetime and curvature and a smaller Hubble constant. We conclude the normalized spacetime parameter, $\Omega_{k}$, should not be ignored and it includes the combined contributions of huge spacetime magnitude and curvature.
\end{abstract}

Keywords Redshift $\cdot$ FRW $\cdot$ Supernova $\cdot$ Planck constant $\cdot$ Emission

\section{Introduction}

The currently popular standard model for spacetime expansion predicts a flat Universe geometry with a large influence of vacuum energy (dark energy) when measured with supernovae type Ia (SNe Ia) data. We previously examined relationships of the Friedmann model with the Robertson-Walker metric (FRW), the standard model, and found discontinuities at low matter densities in our likely future [31, 42]. These discontinuities also influence

\footnotetext{
A.M. Öztaş

Department of Engineering Physics, Hacettepe University, 06800 Ankara, Turkey

M.L. Smith ( $\varangle)$

Anabolic Laboratories, LLC, Tempe, AZ 85281, USA

e-mail: mlsmith10@cox.net

J. Paul

Division of Physics, Luleå University of Technology, 971 87, Luleå, Sweden
} 
the character of the standard model extending to current matter densities and are problematic [24]. To address this we recently proposed a quantitative model describing emissions slowly decreasing in frequency with advancing time while displaying a very slight uncoupling from energy [40]. We did this without knowledge of similar, qualitative proposals by astronomers for emission bluing with increasing lookback time [27]. Another study presented data that light from distant $\mathrm{SNe}$ Ia and resident galaxies are both bluer than recent explosions [15], which hints at a smaller value for Planck's constant in a younger universe.

The history and fate of our expanding Universe has intrigued many people for many decades [25], and for crude solutions, estimates of the average matter density, spacetime curvature and the intergalactic expansion rate are all required. These are only obtained from astronomical observations and recent technical advances have enhanced the data quality. Within the decade, light from $\mathrm{SNe}$ Ia has been used to gauge ancient galactic distances with corresponding redshifts. The SNe Ia result from thermonuclear explosions of Chandrasekhar-mass white dwarves fed by large pools of nearby gases [29]. These are considered good standard candles for distance determination since the explosions are triggered at a unique critical mass with enormous and uniform energy release and predictable spectral evolution, allowing estimates of both distances and spacetime expansion [19]. The data show Hubble's law approximately correct within narrow limits for nearby events and the Universe matter density currently to be well below critical [21].

Researchers have also announced our Universe is expanding at a greater rate than past epochs $[33,34]$. This is unexpected since General Relativity suggests spacetime expansion will gradually slow due to the constant "tug" of gravity. One popular model includes a term for spontaneous energy from the vacuum of outer space, driving a steadily increasing universe expansion; an interesting idea first suggested by Einstein and rekindled by others [7, 8] and fits recent data fairly well $[1,2,10]$. Other models have been suggested to explain the data [27]. The presence of considerable dust between us and older $\mathrm{SNe}$ Ia is one possible explanation for the dimmer, distant supernovae [13] and a version of this dusty explanation fits the data as well as the currently favored standard model [20]. Another explanation may be that the SNe Ia explosion itself has been evolving through time, though thought unlikely by nearly all [19]; such might require violation of $E=m c^{2}$. Initial investigations have been made of possible color evolution of the emission spectrum of SNe Ia; but are inconclusive [11].

An almost universal convention is the historical use of the redshift, $z$ in cosmology, which does not allow full examination of emission frequency models with $\mathrm{SNe}$ Ia data through terms in $z \approx 1$. We address this problem using emission frequency ratios and then derive simple and general solutions for Universe expansion tests according to FRW. Frequency ratio decline is also mentally convenient because it mirrors the spacetime expansion factor. We examine several models using the self-consistent data of 192 SNe Ia distances with associated redshifts $[12,36,44]$ about $z \approx 1$ and find our new model fits the data as well as the current standard model. Models which minimize the normalized spacetime-curvature parameter, $\Omega_{k}$, do not always correlate with the data as well as those which allow a significant value for $\Omega_{k}$. We interpret this to mean that spacetime is very large and while spacetime curvature seems nearly absent some small positive curvature is present, completely consistent with a prediction by Einstein [14].

\section{Theory}

We introduce $\xi$ as the ratio of observed frequency to reference frequency, $\frac{v_{1}}{v_{0}}$, related to the red-shift by $\xi=\frac{1}{1+z}$. This ratio is related to the Hubble constant, $H$, and the spacetime 
expansion factor, $R$, as

$$
H^{2}=\left(\frac{\dot{R}}{R}\right)^{2}=\left(\frac{\dot{\xi}}{\xi}\right)^{2}=\frac{8 \pi G}{3} \rho_{m}+\frac{\Lambda}{3} \pm \frac{k}{R^{2}}
$$

where $G$ is Newton's constant, $\rho_{m}$ is matter with radiation density, $\Lambda$ the cosmic constant and $k$ is the constant of integration, the sign indicating an open, flat or closed $(-, 0,+)$ universe geometry. We refer to published formulations $[7,8]$ which rely on normalization using three parameters where $H_{0}$ is the current Hubble constant for the local Universe with the normalizations as

$$
\Omega_{m}=\frac{8 \pi G}{3 H_{0}^{2}} \rho_{m}, \quad \Omega_{\Lambda}=\frac{\Lambda}{3 H_{0}^{2}}, \quad \Omega_{k}=-\frac{k}{R^{2} H_{0}^{2}}, \quad \text { with } 1=\Omega_{m}+\Omega_{\Lambda}+\Omega_{k} .
$$

For all succeeding arguments $\Omega_{m}$ includes matter, cold dark matter (CDM) and radiation [9], $\Omega_{\Lambda}$ is all forms of dark energy and $\Omega_{k}$ is that portion of a universe involved with spacetime curvature. The equations commonly used are dependent upon $z$ and derived after steps such as substitution for $H_{0} t$, rearrangement, followed by reversing this substitution arriving at one useful example with several variations for a universe without dark energy [7, 12, 36, 44]

$$
D_{L}=\frac{c(1+z)}{H_{0} \sqrt{\left|\Omega_{k}\right|}} \operatorname{sinn}\left\{\sqrt{\left|\Omega_{k}\right|} \int_{0}^{z_{1}} \frac{1}{\sqrt{(1+z)^{2}\left(1+\Omega_{m} z\right)}} d z\right\}
$$

where $\operatorname{sinn}$ is $\sin$ for $\Omega_{k}<0$ and $\sinh$ for $\Omega_{k}>0$ and light speed is $c$. For a flat universe with $\Omega_{k}$ of 0 , but with inclusion of the cosmic constant, one instead uses

$$
D_{L}=\frac{c(1+z)}{H_{0}} \int_{0}^{z_{1}} \frac{1}{\sqrt{(1+z)^{2}\left(1+\Omega_{m} z\right)-z(2+z) \Omega_{\Lambda}}} d z
$$

which must be solved numerically. Equation (3) is cast in a form not requiring integration for evaluation with data [40]

$$
D_{L}=\frac{c(1+z)}{H_{0} \sqrt{\left|\Omega_{k}\right|}} \sinh \left\{2\left(\operatorname{arctanh}\left(\sqrt{\left|\Omega_{k}\right|}\right)-\operatorname{arctanh}\left(\frac{\sqrt{\left|\Omega_{k}\right|}}{\sqrt{1+\Omega_{m} z}}\right)\right)\right\} .
$$

We adapt (5) to terms of only $\xi, \Omega_{m}$ and $\Omega_{k}$ for computerized fitting for a universe without dark energy as

$$
D_{L}=\frac{c}{\xi H_{0} \sqrt{\left|\Omega_{k}\right|}} \sinh \left\{2\left(\operatorname{arctanh}\left(\sqrt{\left|\Omega_{k}\right|}\right)-\operatorname{arctanh}\left(\frac{\sqrt{\left|\Omega_{k}\right|}}{\sqrt{\frac{\Omega_{m}}{\xi}+\Omega_{k}}}\right)\right\} .\right.
$$

Only allowing freedom for the matter density and dark energy parameters, (4) becomes the flat, dark energy model, $\Lambda$ flat because $\Omega_{k}$ is 0 and $\Omega_{\Lambda}$ is written in terms of $\Omega_{m}$

$$
D_{L}=\frac{c}{\xi H_{0}} \int_{\xi_{1}}^{1} \frac{1}{\xi \sqrt{\frac{\Omega_{m}}{\xi}+\left(1-\Omega_{m}\right) \xi^{2}}} d \xi
$$

For evaluating models allowing for matter, dark energy and spacetime curvature, the $\wedge \Omega_{k}$ variation, we use a relationship which must also be integrated

$$
D_{L}=\frac{c}{\xi H_{0} \sqrt{\Omega_{k}}} \sinh \left\{\sqrt{\Omega_{k}} \int_{\xi_{1}}^{1} \frac{1}{\xi \sqrt{\frac{\Omega_{m}}{\xi}+\Omega_{\Lambda} \xi^{2}+\Omega_{k}}} d \xi\right\} .
$$


Detailed derivations of these are presented in Appendix A.

The dark energy model is generalized using an equation of state parameter, $w$. This version of (7) is possible for a flat universe which we present as the $\Lambda$ flat, $w$ model by introducing $1+3 w$ as the exponent of the normalized matter-energy term; the detailed derivation is presented in Appendix B

$$
D_{L}=\frac{c}{\xi H_{0}} \int_{\xi_{1}}^{1} \frac{1}{\xi \sqrt{\frac{\Omega_{m}}{\xi^{(1+3 w)}}+\left(1-\Omega_{m}\right) \xi^{2}}} d \xi .
$$

The above is derived from considering the dynamics of pressure and density evolution in a universe; an analogous relationship with $z$ has been presented, though the cosmic constant term is modified [12, 36, 44].

We derive models of historic importance from a few of the above relationships, remembering that $z=(1 / \xi)-1$. The Hubble relationship is now

$$
D_{L}=\frac{c z}{H_{0}}=\frac{c\left(\frac{1}{\xi}-1\right)}{H_{0}},
$$

and the empty universe as applied to nearby $\mathrm{SNe}$ [35]

$$
D_{L}=\frac{c z\left(1+\frac{z}{2}\right)}{H_{0}} \quad \text { is now } \frac{c\left(1-\xi^{2}\right)}{2 H_{0} \xi^{2}} .
$$

For a flat universe with plenty of matter we can use (7) by replacing $\Omega_{m}$ with 1 and after integration we are left with a wonderfully simple, relationship

$$
D_{L}=\frac{c}{\xi H_{0}} \sinh \left\{\int_{\xi_{1}}^{1} \frac{1}{\xi \sqrt{\frac{1}{\xi}}} d \xi\right\}=\frac{c}{\xi H_{0}} \sinh (1-\xi)
$$

which we designate the E-DS, $\Omega_{m}$ model, for a universe with $\Omega_{m}=1$.

Allowing that distant SNe Ia and galaxy emitters are more blue than current $\mathrm{SNe}$ [40] the relationship for microscopic atomic-photon interactions necessarily becomes $\epsilon=s t_{0} v$, where ş is a tiny new fundamental constant with units of energy and $t_{0}$ is the absolute time from singularity. This suggests the atomic emission frequency is dependent on the local, absolute time as calculated from singularity and the Hubble time may be used as an approximation. Slight variations in emission frequencies due to variations in local time are masked from detection by being but tiny differences of the very large value for the local absolute time and the Heisenberg Uncertainty. We estimate the energy constant ş to be of the order $10^{-44}$ ergs and being incredibly small an exact value may elude better definition for decades to come, being dependent upon more exact values of the Universe age and SNe Ia [40]. Such a small energy constant allows the Planck constant to remain nearly invariant at present, but to slowly decrease with lookback time as observed with SNe Ia.

It is interesting to trace this new relationship back to singularity, for approaching this the Heisenberg Uncertainty diminishes and becomes a certainty at singularity. Logic might demand the reverse also be required; singularity cannot coexist with any uncertainty. So singularity would have appeared as a point-certainty to a god-like observer about 14.5 billion years ago. The contraction of the Heisenberg Uncertainty also means the Planck length near singularity was smaller than current thought suggests, allowing more freedom of thought 
for those interested in the first moments of the Universe. These ideas are consistent with the notion that the exact value of the Planck constant is immaterial [17].

Since neither ş nor $t_{0}$ are known to within $10 \%$ or greater, [40], and we have not a clue of the initial, allowed photon frequencies, we suggest the following relationship to correct for frequency increase with increasing lookback time with only a single unknown [40]

$$
\frac{c}{H_{0}}\left(\xi^{\frac{1}{U}}-1\right)=D_{L_{(v)}} .
$$

Here $U$ is a unitless constant and $D_{L_{(v)}}$ is that portion of the distance expansion due to the absolute time-dependence of emission. Since this weak effect is time-dependent we neglect terms to correct for the effects of matter on time because these have been reported as negligible [27]. If $U$ is large $D_{L_{(v)}}$ is small, which is consistent with a very small and extremely gradual change in emission frequencies in our epoch. We refer to the linear combination of (6) and (13) as the $v$ model and suggest this accounts for the total frequency decline observed from distant emitters, where $D_{F R W}$ is the distance associated with Hubble expansion and $D_{\text {tot }}$ the total expansion distance observed

$$
D_{t o t}=D_{F R W}+D_{L_{(v)}} .
$$

We find the standard FRW model without the cosmic constant, but in linear combination with the correction for emission bluing, fits the data as well as the current standard model, as judged using the Bayesian Information Criteria (BIC). Both models fit the $\mathrm{SNe}$ Ia data better with a local Hubble constant from 62.3 to $67 \mathrm{~km} \mathrm{~s}^{-1} \mathrm{Mpc}^{-1}$ rather than the larger $H_{0}$ of 72 of the Freedman group [18]. The details of our analytical techniques are presented in Appendix C.

\section{Results}

We present the results of calculations using with the most recent and largest SNe Ia data set yet, 192 pairs as reported on-line [12,36, 44]. The actual luminary distances and geometric errors are extracted from the $\log$ data and log errors. We first examine $44 \mathrm{SNe}$ Ia with $\xi>0.90$ to estimate the local Hubble constant. These are the most important data because of the small errors about $D_{L}$ with the results and estimates for goodness of fit from GaussNewton routines given in Table 1. The three historic models are fit with one free parameter (FP) allowing only $H_{0}$ to vary; we allow both $H_{0}$ and $\Omega_{m}$ to vary for the $\Lambda$ flat model; both $H_{0}$ and $U$ to vary for the $\nu \Omega_{m}$ model. The fits were good but not excellent as judged by $\chi^{2} /(N-F P)$ near 1.35 but far from unity [30]. All fits yield values for $H_{0}$ slightly greater than recently $\left(62.3 \mathrm{~km} \mathrm{~s}^{-1} \mathrm{Mpc}^{-1}\right)$ published [38] but smaller than the result (72) of the Freedman group [18]. Among the historic models the empty universe fit best and the Hubble and E-DS models yield similar $H_{0}$ and $\chi^{2}$. The good fit for the $\nu \Omega_{m}$ model is deceiving, for the routine allows $U$ to become incredibly large and unimportant, so the $\nu \Omega_{m}$ model mimics the empty universe. The $\Lambda$ flat fit is also deceiving because $\Omega_{m}$ is allowed to vary, becoming negligible and hence another variation of the empty universe model, but filled with dark energy. We note the best values for the Hubble constant—in the mid 60s—will return again as the best values using all the data with more advanced models. Clearly data for $\xi \ll 0.90$ are necessary to discriminate between simple and sophisticated universes and most simple model fits prefer an empty universe. 
Table 1 Results from "nearby" 44 SNe Ia with $\xi>0.90$ for historic models

\begin{tabular}{llllll}
\hline Model & $H_{0}\left(\mathrm{~km} \mathrm{~s}^{-1} \mathrm{Mpc}^{-1}\right)$ & Error & $\chi^{2}$ & $\chi^{2} /(N-F P)$ & $\Delta B I C$ \\
\hline Hubble & 64.6 & 1.0 & 59.6 & 1.39 & 2.4 \\
E-DS, $\Omega_{m}=1.0$ & 64.6 & 1.0 & 59.5 & 1.38 & 2.3 \\
$\nu \Omega_{m}{ }^{*}$ & 65.3 & 2.0 & 56.9 & 1.35 & 5.4 \\
empty, $\Omega_{m}=0$ & 65.7 & 1.0 & 56.5 & 1.31 & 0 \\
$\Lambda$ flat $^{*}$ & 66.7 & 2.0 & 54.7 & 1.30 & 3.8 \\
\hline
\end{tabular}

*With $\Omega_{m}$ of 0.20 , allowing both $H_{0}$ and $U$ to vary for the $\nu \Omega_{m}$ model; $H_{0}$ and $\Omega_{m}$ to vary for the $\Lambda$ flat model; E-DS, Einstein-DeSitter model. Errors are standard deviations, $N$ the number of data pairs and FP the number of free parameters (either 1 or 2)

Table 2 Results using all $192 \mathrm{SNe}$ Ia, varying $H_{0}$ stepwise with robust fitting and one free parameter

\begin{tabular}{lllllllllll}
\hline Model & $H_{0}$ & $\Omega_{m}$ & Error & $\Omega_{k}$ & Error & $U$ & Error & $\chi^{2}$ & $\chi^{2} /(N-1)$ & $\Delta B I C$ \\
\hline$\Lambda \Omega_{k}$ & 67 & 0.27 & fixed & $-0.003^{*}$ & 0.011 & - & - & 214.3 & 1.12 & 0 \\
$\Lambda$ flat & 67 & $0.23^{*}$ & 0.02 & - & - & - & - & 215.2 & 1.13 & 1.4 \\
$\nu \Omega_{m}$ & 62.3 & 0.07 & fixed & 0.93 & fixed & $715^{*}$ & 5136 & 238.5 & 1.25 & 21.1 \\
$\Lambda$ flat & 62.3 & $0.48^{*}$ & 0.03 & - & - & - & - & 244.4 & 1.28 & 26.8 \\
$\nu \Omega_{m}$ & 67 & 0.06 & fixed & 0.94 & fixed & $13^{*}$ & 2 & 252.2 & 1.32 & 31.2 \\
\hline
\end{tabular}

*Free parameter, errors are standard deviations and $N$ the number of data pairs (192)

We examine all 192 data pairs (Table 2) allowing one FP; the $\chi^{2}$ are larger than reported by Astier et al. [1], the results of fitting the data directly, not as $\log$ (values) and because of this our robust calculations also place greater reliance on data from recent SNe. We examine both the dark energy and blue light models taking $H_{0}$ in steps of 1 , from 72 to $62.3 \mathrm{~km} \mathrm{~s}^{-1} \mathrm{Mpc}^{-1}$. All of these fits exhibit low $\chi^{2}$ with $\chi^{2} /(N-1)$ well below the value of 1.35 and should be considered good [30]. The dark energy model for a nearly flat universe $\left(\Omega_{m}+\Omega_{\Lambda}+\Omega_{k}=1\right)$ with an $H_{0}$ of 67 fit the data best with a fixed, normalized matter density of 0.27 and a slightly open universe $\left(\Omega_{k}=-0.003\right)$. When $\Omega_{m}$ is allowed to float $\left(\Omega_{m}+\Omega_{\Lambda}=1\right.$ and $\left.\Omega_{k}=0\right)$ at this Hubble constant the matter density declines to 0.23 ; both values for $\Omega_{m}$ are within the current range popular with many astronomers. When a Hubble constant of 62.3 is used with the flat, dark energy model the surprising result for $\Omega_{m}$ of 0.48 is found but with a small error of 0.03 ; an error similar to other reports [1]. We also examine a universe fixing $\Omega_{m}$ at the popular value of $0.27, H_{0}$ at $62.3 \mathrm{~km} \mathrm{~s}^{-1} \mathrm{Mpc}^{-1}$ and allow the combination of $\Omega_{\wedge}$ and $\Omega_{k}$ to vary as one FP. Under these restraints the model fit well, though $\Omega_{\wedge}$ is reduced from the value of 0.73 to 0.53 and $\Omega_{k}$ is a significant 0.20 . For the $v \Omega_{m}$ model we use $\Omega_{m}+\Omega_{k}=1$, allow freedom only for $U$ and examine solutions with $\Omega_{m}$ fixed in 0.01 steps, between 0.01 and 0.30 . This is necessary because the two terms discourage meaningful examination with only $1 \mathrm{FP}$, unless this is $H_{0}$. While the goodness of fit is better for the $\Lambda$ flat model towards high matter densities, the reverse is true for the $\nu \Omega_{m}$ model where the best fit is observed at $\Omega_{m}=0.07$. The values for $U$ of 715 is reasonable since a $U$ up to $10^{6}$ is within the range of detection using lookback data [41]. Unfortunately, the error for $U$ is large since this constant appears as a fractional exponent in the solution, compounding the effect of being a weak addition on top of the strong redshift due to Universe expansion. This large error is also evidence that fit minima with one 
Table 3 Results using all $192 \mathrm{SNe}$ Ia, varying $H_{0}$ stepwise with robust fitting and two free parameters

\begin{tabular}{lllllllllll}
\hline Model & $H_{0}$ & $\Omega_{m}$ & Error & $\Omega_{\Lambda}$ & $w$ & $U$ & Error & $\chi^{2}$ & $\chi^{2} /(N-2)$ & $\Delta B I C$ \\
\hline$\Lambda$ flat,$w$ & 67 & $0.11^{*}$ & 0.05 & 0.89 & $0.46^{*}$ & - & 0.33 & 214.2 & 1.13 & 0.0 \\
$\Lambda \Omega_{k}$ & 67 & $0.23^{*}$ & 0.14 & $0.77^{*}$ & - & - & 0.14 & 215.4 & 1.13 & 0.2 \\
$\nu \Omega_{k}$ & 62.3 & $0.09^{*}$ & 0.0 & - & - & $19^{*}$ & 5 & 217.1 & 1.14 & 2.2 \\
$\nu \Omega_{k}$ & 67 & $0.03^{*}$ & 0.0 & - & - & $7.5^{*}$ & 0.9 & 218.0 & 1.15 & 2.1 \\
\hline
\end{tabular}

*Free parameter, errors are standard deviations and $N$ the number of data pairs (192)

FP for $\nu \Omega_{m}$ models are fairly shallow. When we use a $H_{0}$ of 67 , the best value for the $\Lambda$ models, the best fit for the $\nu \Omega_{m}$ model was at an even lower matter density, 0.06 , while $U$ becomes small and important. As a comparison we fit the model for the empty universe, (11) with one FP and observe a fair fit with a $\chi^{2}$ of 260.7 and a $\chi^{2} /(N-1)$ of 1.36 ; a borderline good fit. Here $H_{0}$ is found to be $66.3 \pm 0.06 \mathrm{~km} \mathrm{~s}^{-1} \mathrm{Mpc}^{-1}$; near the mean between the values from Sandage et al. [38] and Freedman et al. [18]. We suggest the large $\Delta$ BIC between the two $\Lambda$ models and the two $\nu \Omega_{m}$ models are primarily due to the requirement that $\Omega_{m}$ and $\Omega_{k}$ remain fixed for single parameter fits of the $v \Omega_{m}$ models. We also note the larger value for the Hubble constant (72) did not yield very good fits with any model.

We next examine three interesting models allowing two FP, $\Omega_{m}$ and $w$ for the $\Lambda$ flat, $w$ model $\left(\Omega_{m}+\Omega_{\Lambda}=1\right), \Omega_{\Lambda}$ and $\Omega_{k}$ for the $\Lambda \Omega_{k}$ model $\left(\Omega_{m}+\Omega_{\Lambda}+\Omega_{k}=1\right)$, and the $\Omega_{k}$ and $U$ for the $v \Omega_{k}$ model $\left(\Omega_{m}+\Omega_{k}=1\right)$, with results in Table 3. All models fit the data quite well as judged by $\chi^{2} /(N-2)$ values and with differences in BIC being less than 6 there is no reason for model rejection by statistics. While the $\Lambda$ flat, $w$ model fit best, the free parameter $w$ is found far removed from the default value of -1 and the matter density falls towards the low value expected of a model rich in dark energy or spacetime density. The $\Lambda$ models fit the data no better than allowing only $1 \mathrm{FP}$ and not significantly better than the $\nu \Omega_{k}$ models as judged by $\chi^{2}$ and BIC. The $\Lambda \Omega_{k}$ model remains nearly flat with $\Omega_{k}$ of $\approx 0.00$ despite the extra FP. We find the $\Lambda \Omega_{k}$ model interesting because $\Omega_{m}$ increases drastically to 0.53 and $\Omega_{\Lambda}$ decreases to 0.47 when a $H_{0}$ of 62.3 is used and the errors for both $\Omega_{m}$ and $\Omega_{\Lambda}$ become large (results not presented). Even with Hubble constant of 67, yielding the best fits, the residuals about the values for the matter and dark energy densities are rather large. The goodness of fit is better at lower matter density for the $\nu \Omega_{k}$ model than the $\Lambda$ models. The $\nu \Omega_{k}$ model, now with genuine $2 \mathrm{FP}$, fits the data well with small error about $\Omega_{m}$ and a reasonable $U$ between 7 and 19. In a separate evaluation of the $\nu \Omega_{k}$ model we fixed $\Omega_{m}$ at 0.20 , with $\Omega_{m}+\Omega_{k}=1$, allowing $H_{0}$ and $U$ to be the two FP. With a $\chi^{2}$ of $244.7, H_{0}$ was calculated as $62.2 \pm 2.1 \mathrm{~km} \mathrm{~s}^{-1} \mathrm{Mpc}^{-1}$ with $U$ of $1804 ; H_{0}$ is close to that of Sandage and coworkers [38].

A trace of the $\Lambda$ flat, $w$ model is shown in Fig. 1 being moved more towards frequency decline compared with the other two traces. With so much data and such large errors it seems impossible to prefer one model over another by visual examination of this plot. Figure 2 presents the calculated residuals for the $\nu \Omega_{k}$ and the $\Lambda \Omega_{k}$ models with 2 FP from Fig. 1 . These appear almost interchangeable with the residuals from the most recent $\mathrm{SNe}$ Ia tightly concentrated about the best fit towards the right side of the plots, but widening drastically with increasing $z$. 


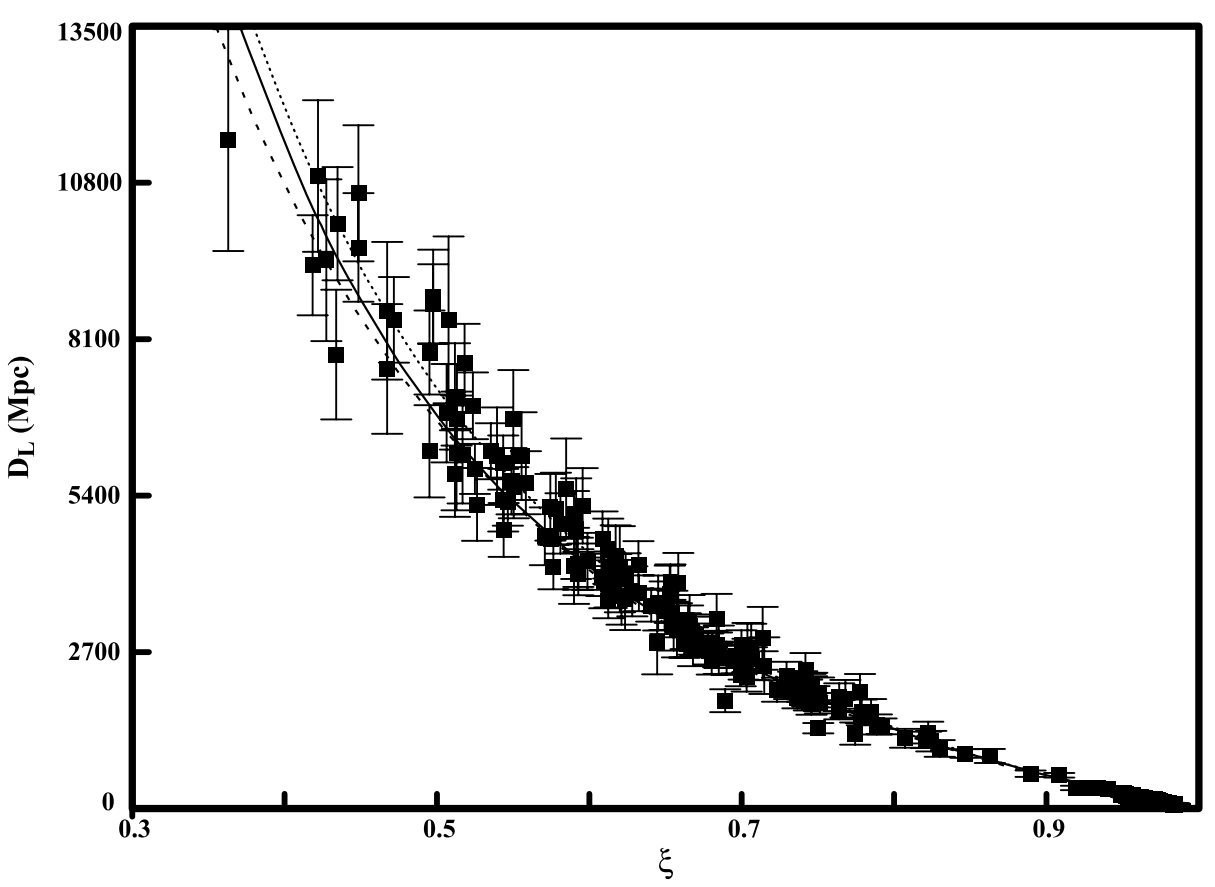

Fig. 1 Three models with all SNe Ia data fit with $H_{0}$ of $62.3 \mathrm{~km} \mathrm{~s}^{-1} \mathrm{Mpc}^{-1}$ : left, dashed $\Lambda \Omega_{k}$; middle, line $\nu \Omega_{k} ;$ right, dotted $\Lambda$ flat, $w$
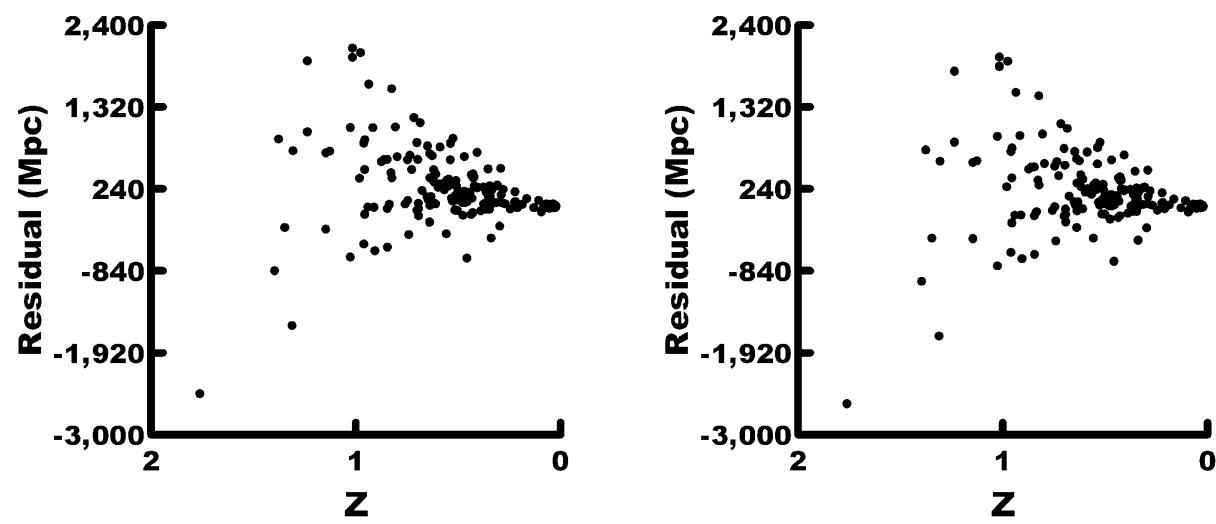

Fig. 2 Residuals verses redshift ( $z$ used for display only) for models allowing two free parameters with $H_{0}$ of $62.3 \mathrm{~km} \mathrm{~s}^{-1} \mathrm{Mpc}^{-1}:$ left,$\Lambda \Omega_{k}$, and right, $\nu \Omega_{k}$. Note the data exhibiting small residuals are located toward small $z$ and the standard errors for some data at large $z$ may be more than a billion years

\section{Discussion}

We present useful relationships for modeling the expanding universe in terms of distances and emission frequency decline with the FRW conditions, allowing continuous solutions that include terms about $z \approx 1(\approx 5-6$ Gyears ago). Though using the cosmic constant with a simple equation of state $(w=-1)$ and a flat universe is currently popular, other models 
are worth considering. For instance, one model conveniently folds the Hubble expansion into General Relativity with the benefit of correctly predicting important properties of our Universe [4]. This model straightforwardly predicts a cold dark matter (CDM)-free, TullyFisher relationship [6, 22]. The best solution with earlier published SNe Ia data and that model is found at low matter density $\left(\Omega_{m}=0.021\right)$ so does not require CDM or even dark energy $[23,30]$ but does conform nicely to some historical measurements of $\Omega_{m}$ below 0.05 [16] and to recent estimates of low baryonic density from the big bang nucleosynthesis [5]. The best fits for the models considered here might also suggest that CDM and dark energy are not terribly important and that our Universe consists mainly of spacetime.

The general tendency of $\Lambda$ models are to remain flat and predict high matter density while the tendency of the blue emission, $v$, model is to hedge towards an empty universe with plenty of curved spacetime. This tendency of the $\Lambda$ models may be an artifact of mathematics because models depending on significant dark energy display discontinuities at low matter densities [31] and the effect is propagated towards lower matter densities considered here. Models allowing a slight variation of the Planck constant fit current $\mathrm{SNe}$ Ia data at least as well, considering $\chi^{2} /(N-2)$ values approaching 1 and the BIC values equivalent to the standard model. The models examined here did not fit well with the larger $H_{0}$ of $72 \mathrm{~km} \mathrm{~s}^{-1} \mathrm{Mpc}^{-1}$ but with values between 62.3 and 67.

Because $\chi^{2} /(N-2)$ and BIC are similar between models when fit using two FP, we think detection of significant differences will require another two hundred $\mathrm{SNe}$ data pairs, but only if the $\mathrm{S} / \mathrm{N}$ ratio can be increased 3 -fold. This will require a 10 -fold increase in observational time for each $\mathrm{SN}$, but if obtained, the $\triangle$ BIC between models may increase to about 12 , which should be considered significant. The collection of more SNe Ia data as a test of the $v$ model might also be our best opportunity for checking the fundamental nature of the Planck constant and someday allow an accurate estimate of $t_{0}, U$ and questions of the hyperfine constant [3]. Benchtop measurements of the Planck constant are ongoing but do not seem accurate or precise enough to record such a tiny drift as predicted here [37]. A recent monograph presents another 10 constants of interest, some which might be investigated using SNe Ia data [28]. Using $D_{L}$ and $z$ rather than enormous energies may be the best method for finding deeper components for some of these values.

The models examined also differ in physics. The $\Lambda$ model does not correlate very well with calculated energy from microscopic vacuum fluctuations and is far removed from concordance with Newton's $G$ [8]. Dark energy seems a case for physics operating only on galactic dimensions but not atomic scales, which may be true but difficult to test on earth and also demands more fundamental thought. On the other hand, blue emissions have been observed from both exploding $\mathrm{SNe}$ Ia and associated galaxies and may be a general effect [15] and the expected value for $U$ is within observation when gauged against the Universe age and first star emission [41]. Such small bluing would also not affect the physics of recombination after singularity, only the timing of this event with respect to the present epoch; of practical importance for those studying galaxy and star formation. The most realistic models, here and by others [23] are found to include a significant contribution from spacetime-curvature, $\Omega_{k}$. We interpret this first to mean we are a long time removed from the epoch of critical mass density, $\Omega_{m} \approx 1$, and then to mean this parameter indicates contributions both from spacetime, which is obviously enormous, and curvature, which seems tiny. 


\section{Appendix A}

We will briefly derive the dependence of luminar distances on the frequency ratio from the conditions of the FRW model. The first derivative of the frequency ratio with respect to time is related to the redshift increase by

$$
\dot{\xi}=\frac{-\dot{z}}{(1+z)^{2}}
$$

This allows us to write the Friedmann relationships in a straightforward manner as

$$
H^{2}=\left(\frac{\dot{R}}{R}\right)^{2}=\left(\frac{-\dot{z}}{1+z}\right)^{2}=\frac{\dot{\xi}^{2}}{\xi^{2}} .
$$

It is convenient to introduce the normalized matter, vacuum energy and spacetime curvature from (16) into (15), to derive a relationship with these three constants and the local Hubble constant

$$
\frac{\dot{\xi}^{2}}{\xi^{2}}=H_{0}^{2} \frac{\Omega_{m}}{\xi^{3}}+H_{0}^{2} \Omega_{\Lambda}+H_{0}^{2} \frac{\Omega_{k}}{\xi^{2}} .
$$

We now introduce the parameter $\tau$ for $H_{0} / \xi$ into (17) to yield

$$
\left(\frac{d \xi}{d \tau}\right)^{2}=\frac{\Omega_{m}}{\xi}+\Omega_{\Lambda} \xi^{2}+\Omega_{k}
$$

Then solving for $d \tau$ we find

$$
d \tau=\frac{d \xi}{\sqrt{\frac{\Omega_{m}}{\xi}+\Omega_{\Lambda} \xi^{2}+\Omega_{k}}} .
$$

A Einstein-DeSitter universe (E-DS) may be solved by letting $\Omega_{\Lambda}=0$, and $\Omega_{m}+\Omega_{k}=1$. We refer the reader to [32] for substitutions on the left-hand side of (19), and by dropping the middle term in the denominator leads us to a relationship for a universe without dark energy

$$
H_{0} R_{0} \frac{d r}{\sqrt{1+\Omega_{k} H_{0}^{2} R_{0}^{2} r^{2}}}=\frac{d \tau}{\xi}=\frac{1}{\xi} \frac{d \xi}{\sqrt{\frac{\Omega_{m}}{\xi}+\Omega_{k}}} .
$$

Both sides can be integrated from the past to the present because we have separated distance from $\xi$

$$
H_{0} R_{0} \int_{0}^{r_{1}} \frac{1}{\sqrt{1+\Omega_{k} H_{0}^{2} R_{0}^{2} r^{2}}} d r=\int_{\xi_{1}}^{1} \frac{1}{\xi \sqrt{\frac{\Omega_{m}}{\xi}+\Omega_{k}}} d \xi .
$$

Integration of the left-hand side of (21) leaves us with an analytical term and the right-hand side can also be solved. We continue by using $y=\frac{\Omega_{m}}{\xi}+\Omega_{k}$ which allows us to substitute for

$$
\xi=\frac{\Omega_{m}}{\left(y^{2}-\Omega_{k}\right)} \quad \text { and } \quad d \xi=-2 \frac{\Omega_{m} y}{\left(y^{2}-\Omega_{k}\right)^{2}}
$$


which leads us to

$$
\frac{1}{\sqrt{\Omega_{k}}} \operatorname{arcsinh}\left(\sqrt{\Omega_{k}} R_{0} H_{0} r_{1}\right)=2 \int \frac{1}{y^{2}-\Omega_{k}} d y .
$$

When this integral is evaluated from $\sqrt{\frac{\Omega_{m}}{\xi}+\Omega_{k}}$ to 1 we derive the analytical solution for the E-DS universe in terms of the $\xi, \Omega_{m}$ and $\Omega_{k}$

$$
\operatorname{arcsinh}\left(\sqrt{\Omega_{k}} R_{0} H_{0} r_{1}\right)=2\left(\operatorname{arctanh}\left(\frac{1}{\sqrt{\Omega_{k}}}\right)-\operatorname{arctanh}\left(\frac{\sqrt{\frac{\Omega_{m}}{\xi}+\Omega_{k}}}{\sqrt{\Omega_{k}}}\right)\right) .
$$

We now reverse the substitutions of the left-hand side using the astronomical relation $D_{M}=R_{0} r_{1}$

$$
\sqrt{\Omega_{k}} H_{0} D_{M}=\sinh \left\{2\left(\operatorname{arctanh}\left(\frac{1}{\sqrt{\Omega_{k}}}\right)-\operatorname{arctanh}\left(\frac{\sqrt{\frac{\Omega_{m}}{\xi_{1}}+\Omega_{k}}}{\sqrt{\Omega_{k}}}\right)\right)\right\}
$$

then by substituting for $D_{M} / \xi$ with $D_{L}$, introducing $c$ for the speed of light and using the relationship of $\operatorname{arctanh}(a)-\operatorname{arctanh}(b)=\operatorname{arctanh}(1 / a)-\operatorname{arctanh}(1 / b)$ to invert some of the terms we arrive at a very useful formula

$$
D_{L}=\frac{c}{\xi H_{0} \sqrt{\Omega_{k}}} \sinh \left\{2\left(\operatorname{arctanh}\left(\sqrt{\Omega_{k}}\right)-\operatorname{arctanh}\left(\frac{\sqrt{\Omega_{k}}}{\sqrt{\frac{\Omega_{m}}{\xi_{1}}+\Omega_{k}}}\right)\right)\right\} .
$$

Unfortunately, the relationship including the cosmic constant cannot be solved analytically and we shall proceed from (19) to a form which may be integrated by computer, so we reintroduce the term including the cosmic constant

$$
H_{0} R_{0} \frac{d r}{\sqrt{1+\Omega_{k} H_{0}^{2} R_{0}^{2} r^{2}}}=\frac{d \tau}{\xi}=\frac{1}{\xi} \frac{d \xi}{\sqrt{\frac{\Omega_{m}}{\xi}+\Omega_{\Lambda} \xi^{2}+\Omega_{k}}}
$$

and integrate both sides for distance and frequency ratio to the present

$$
H_{0} R_{0} \int_{0}^{r_{1}} \frac{1}{\sqrt{1+\Omega_{k} H_{0}^{2} R_{0}^{2} r^{2}}} d r=\int_{\xi_{1}}^{1} \frac{1}{\xi \sqrt{\frac{\Omega_{m}}{\xi}+\Omega_{\Lambda} \xi^{2}+\Omega_{k}}} d \xi .
$$

This time we can only integrate the left-hand side of the equation

$$
\sqrt{\Omega_{k}} R_{0} H_{0} r_{1}=\sinh \left\{\sqrt{\Omega_{k}} \int_{\xi_{1}}^{1} \frac{1}{\xi \sqrt{\frac{\Omega_{m}}{\xi}+\Omega_{\Lambda} \xi^{2}+\Omega_{k}}} d \xi\right\} .
$$

By substituting again for $R_{0} r_{1}$ and then again for $D_{M}$ and inserting $c$ for light speed we arrive at

$$
D_{L}=\frac{c}{\xi H_{0} \sqrt{\Omega_{k}}} \sinh \left\{\sqrt{\Omega_{k}} \int_{\xi_{1}}^{1} \frac{1}{\xi \sqrt{\frac{\Omega_{m}}{\xi}+\Omega_{\Lambda} \xi^{2}+\Omega_{k}}} d \xi\right\}
$$

which we refer to as the $\Lambda \Omega_{k}$ model. The absolute value for $\Omega_{k}$ is always used for calculations; convenient because this parameter is sometimes allowed to vary during computerized fits. 


\section{Appendix B}

We begin with two equations of state from [32, 5.15 and 5.17], describing our Universe

$$
\begin{aligned}
\left(\frac{\dot{a}}{a}\right)^{2} & =\frac{8 \pi G}{3} \rho+\frac{\Lambda}{3}+\frac{k}{a^{2}}, \\
\frac{\ddot{a}}{a} & =\frac{-4 \pi G}{3}(\rho+3 p)+\frac{\Lambda}{3} .
\end{aligned}
$$

Here $\rho$ is the density of material items and $3 p$ the current Universe pressure with the other symbols as previously introduced. For the general situation both variables include contributions from normal matter, radiation and cold dark matter (though CDM supposedly adds to the density but perhaps not the pressure?). We then take the derivative of the first equation with respect to time and use the result to eliminate $\ddot{a}$ and arrive at the following relationship

$$
\dot{\rho}=-3 \frac{\dot{a}}{a}(\rho+p)
$$

We presume pressure a simple, linear function of density, with $p=w \rho$, and we proceed attempting a solution in terms of $\xi$ and $D_{L}$ beginning with simple substitutions

$$
\begin{aligned}
\dot{\rho} & =-3 \frac{\dot{a}}{a} \rho(1+w), \\
\frac{\dot{\rho}}{\rho} & =-3(1+w) \frac{\dot{a}}{a}, \\
\int \frac{\dot{\rho}}{\rho} d t & =-3(1+w) \int \frac{\dot{a}}{a} d t, \\
\ln (\rho) & =-3(1+w) \ln (a)+\ln \left(\rho_{0}\right), \\
\rho & =\rho_{0} a^{-3(1+w)}
\end{aligned}
$$

and we designate $\rho_{0}$ to be the present value of all densities, combined. Since the Universe consists of different species of matter and energies, the density consists of several components [26] which may be collected as

$$
\rho=\sum_{i}^{N} \frac{\rho_{i, 0}}{a^{3(1+w)}} .
$$

These are defined in a normalized manner for the more well-known varieties of matter and energy as

$$
\Omega_{i, 0}=\frac{8 \pi G}{3 H_{0}} \rho_{i, 0}
$$

and as we recast the FRW relationship, using this normalization, we shall begin with

$$
\left(\frac{\dot{a}}{a}\right)^{2}=H_{0}^{2}\left(\sum_{i}^{N} \frac{\Omega_{i, 0}}{a^{3\left(1+w_{i}\right)}}+\Omega_{\Lambda}+\frac{\Omega_{k}}{a^{2}}\right)
$$


where $w_{i}$ are the various linear relationships between densities. When we substitute $\tau$ for $H_{0} / a$ we can simplify this equation slightly with the normalization between terms as given below

$$
\begin{aligned}
\left(\frac{d a}{d \tau}\right)^{2} & =\sum_{i}^{N} \frac{\Omega_{i, 0}}{a^{\left(1+3 w_{i}\right)}}+\Omega_{\Lambda} a^{2}+\Omega_{k}, \\
1 & =\sum_{i}^{N} \Omega_{i, 0}+\Omega_{\Lambda}+\Omega_{k} .
\end{aligned}
$$

We introduce the frequency decline being directly proportional to spacetime expansion, in terms of $\tau$ and this normalization becomes

$$
\frac{d \xi}{d \tau}=\sqrt{\sum_{i}^{N} \Omega_{i, 0}+\Omega_{\Lambda}+\Omega_{k}}
$$

and with rearrangement we reintroduce $w$

$$
d \tau=\frac{d \xi}{\sqrt{\sum_{i}^{N} \frac{\Omega_{i, 0}}{\xi^{\left(1+3 w_{i}\right)}}+\Omega_{\Lambda} \xi^{2}+\Omega_{k}}} .
$$

We simplify the situation by limiting the solution to the sum of common and CDM, ignoring the miniscule contribution of radiation leading to

$$
H_{0} D_{L} \sqrt{\Omega_{k}}=\frac{c}{\xi} \sinh \left(\sqrt{\Omega_{k}} \int_{\xi_{1}}^{1} \frac{1}{\xi \sqrt{\sum_{i}^{N} \frac{\Omega_{i, 0}}{\xi^{\left(1+3 w_{i}\right)}}+\Omega_{\Lambda} \xi^{2}+\Omega_{k}}} d \xi\right)
$$

For a flat universe $\left(\Lambda\right.$ flat,$w$ model) we substitute $1-\Omega_{m}$ for $\Omega_{\Lambda}$ and ignore the contributions of $\Omega_{k}$

$$
D_{L}=\frac{c}{\xi H_{0}} \int_{\xi_{1}}^{1} \frac{1}{\xi \sqrt{\sum_{i}^{N} \frac{\Omega_{i, 0}}{\xi^{\left(1+3 w_{i}\right)}}+\left(1-\Omega_{m}\right) \xi^{2}}} d \xi
$$

This is the our (9) with results in Table 3.

\section{Appendix C}

Luminar distances, $D_{L}$ in terms of $\mathrm{Mpc}$, are calculated from the distance moduli using the formula

$$
D_{L}=10^{\left(\mu_{p}-25\right) / 5}
$$

We employ distances and geometric errors of the distances, here termed errors, for curve fitting calculated from the log-log data available on-line [12, 36, 44]. These are highly dependent on absolute distances measured from nearby Cepheid variable stars; internal selfconsistency is claimed. The largest portions of Cepheid distance errors are thought systematic [38]. 
Robust, non-linear least squared fitting is used with all $192 \mathrm{SNe}$ Ia data pairs back to $\xi$ of 0.363 . In separate calculations, 44 data pairs with values for $\xi>0.90$ are used with the Gauss-Newton method; the "Hubble" portion of the curves. The best fits from the lowest $\chi^{2}$ values calculated using the common relationship [43]

$$
\chi^{2}=\sum_{i} \frac{\left(s_{D_{L}, i}\right)^{2}}{\sigma_{D_{L}, i}^{2}}
$$

are presented, where $\sigma_{D_{L}, i}$ are the estimated uncertainties in the individual distance observations and $s_{D_{L}, i}$ are the calculated individual errors from the fit. Values for $\sigma_{D_{L}, i}$ contain some degree of uncertainty due to galaxy peculiar velocities which have been added by those authors; this contribution should be minor because only data from SNe Ia with $z>0.0160$ (230 Myears distant) have been selected. Since we use the data as Mpc rather than the $\log (\mathrm{Mpc})$ our $\chi^{2}$ are larger than those typically reported and the results are more dependent on recent SNe Ia events with smaller errors rather then distant SNe Ia (larger errors). Many of our calculations are based upon a $H_{0}$ of $62.3 \mathrm{~km} \mathrm{~s}^{-1} \mathrm{Mpc}^{-1}$ [38] using starting parameter estimates near and far from expectations.

For model comparisons we apply the Bayesian information criteria (BIC) formulated as

$$
B I C=-2 \ln \mathcal{L}+k \ln N
$$

according to [39] where $\mathcal{L}$ is the maximum likelihood, $k$ the number of parameters fit and $N$ the number of data pairs. When the errors are presumed Gaussian this criteria becomes

$$
B I C=N \ln \left(\frac{S S E}{N}\right)+k \ln N
$$

where $S S E$ is the sum of squared errors calculated from curve-fitting. The BIC is useful to compare fits when different number of parameters are evaluated and the lower the BIC the better the model. We present our $\triangle B I C$ as relative values.

\section{References}

1. Astier, P., Guy, J., Regnault, N., Pain, R., Aubourg, E., Balam, D., Basa, S., Carlberg, R.G., Fabbro, S., Fouchez, D., Hook, I.M., Howell, D.A., Lafoux, H., Neill, J.D., Palanque-Delabrouille, N., Perrett, K., Pritchet, C.J., Rich, J., Sullivan, M., Taillet, R., Aldering, G., Antilogus, P., Arsenijevic, V., Balland, C., Baumont, S., Bronder, J., Courtois, H., Ellis, R.S., Filiol, M., Goncalves, A.C., Goobar, A., Guide, D., Hardin, D., Lusset, V., Lidman, C., McMahon, R., Mouchet, M., Mourao, A., Perlmutter, S., Ripoche, R., Tao, C., Walton, N.: Astron. Astrophys. 447, 31 (2006)

2. Barris, B.J., Tonry, J.L., Blondin, S., Challis, P., Chornock, R., Clocchiatti, A., Filippenko, A.V., Garnavich, P., Holland, T., Jha, S., Kirshner, R.P., Krisciunas, K., Leibundgut, B., Li, W., Matheson, T., Miknaitis, G., Riess, A.G., Schmidt, B.P., Smith, R.C., Sollerman, J., Spyromilio, J., Stubbs, C.W., Suntzeff, N.B., Aussel, H., Chambers, K.C., Connelley, M.S., Donovan, D., Henry, J.P., Kaiser, N., Liu, M.C., Martin, E.L., Wainscoat, R.J.: Astrophys. J. 602, 571 (2004)

3. Barrow, J.D.: Philos. Trans. R. Soc. Lond. A 363, 2139 (2005)

4. Behar, S., Carmeli, M.: Int. J. Theor. Phys. 39, 1375 (2000)

5. Burles, S., Nollett, K.M., Turner, M.S.: Astrophys. J. 552, L1 (2001)

6. Carmeli, M.: Int. J. Theor. Phys. 39, 1397 (2000)

7. Carroll, S.M., Press, W.H., Turner, E.L.: Annu. Rev. Astron. Astrophys. 30, 499 (1992)

8. Carroll, S.M.: Living reviews in relativity 4. Online Article: www.livingreviews.org/Articles/Volume4/ 2001-1carroll/ Max Planck Institute for Gravitational Physics, Germany (2001)

9. Carroll, S.M.: Nature Physics 2, 653 (2006) 
10. Clocchiatti, A., Schmidt, B.P., Filippenko, A.V., Challis, P., Coil, A.L., Covarrubias, R., Diercks, A., Garnavich, P., Germany, L., Gilliland, R., Hogan, C., Jha, S., Kirshner, R.P., Leibundgut, B., Leonard, D., Li, W., Matheson, T., Phillips, M.M., Prieto, J.L., Reiss, D., Riess, A.G., Schommer, R., Smith, R.C., Soderberg, A., Spyromilio, J., Stubbs, C., Suntzeff, N.B., Tonry, J.L., Woudt, P.: Astrophys. J. 642, 1 (2006)

11. Coil, A.L., Matheson, T., Filippenko, A.V., Leonard, D.C., Tonry, J., Riess, A.G., Challis, P., Clocchiatti, A., Garnavich, P.M., Hogan, C.J., Jha, S., Kirshner, R.P., Leibundgut, B., Phillips, M.M., Schmidt, B.P., Schommer, R.A., Smith, R.C., Soderberg, A.M., Spyromilio, J., Stubbs, C., Suntzeff, N.B., Woudt, P.: Astrophys. J. 544, L111 (2000)

12. Davis, T.M. et al.: astro-ph/0701510 (2007). www.ctio.noao.edu/

13. Drell, P.S., Loredo, T.J., Wasserman, I.: Astrophys. J. 530, 593 (2000)

14. Einstein, A.: Relativity. Routledge Classics, pp. 115-116. Taylor Francis Group, London (1993),

15. Falco, E.E., Imprey, C.D., Kochanek, C.S., Lehar, J., McLeod, B.A., Rix, H.-W., Keeton, C.R., Munoz, J.A., Peng, C.Y.: Astrophys. J. 523, 617 (1999)

16. Fall, S.M.: Mon. Not. R. Astron. Soc. 172, 23 (1975)

17. Finkelstein, D.R.: Quantum Relativity, pp. 163-166. Springer, Heidelberg (1996),

18. Freedman, W.L., Madore, B.F., Gibson, B.K., Ferrarese, L., Kelson, D.D., Sakai, S., Mould, J.R., Kennicutt, Jr. R.C., Ford, H.C., Graham, J.A., Huchra, J.P., Hughes, S.M.G., Illingworth, G.D., Macri, L., Stetson, P.B.: Astrophys. J. 553, 47 (2001)

19. Gallagher, J.S., Garnavich, P.M., Berlind, P., Challis, P., Jha, S., Kirshner, R.P.: Astrophys. J. 634, 210 (2005)

20. Goobar, A., Bergstrom, I., Mortsel, E.: Astron. Astrophys. 384, 1 (2002)

21. Hamuy, M., Phillips, M.M., Maza, J., Suntzeff, N.B., Schommer, R.A., Aviles, R.: Astron. J. 109, 1 (1995)

22. Hartnett, J.G.: Int. J. Theor. Phys. 45, 2147 (2006)

23. Hartnett, J.G., Oliveira, F.J.: Found. Phys. 37, 446 (2007)

24. Hogan, J.: Nature 446, 709 (2007)

25. Hubble, E.: Proc. Nat. Acad. Sci. 15, 168 (1929)

26. Lahav, O., Suto, Y.: Living reviews in relativity 8. Online Article: http://relativity.livingreviews.org/ Articles/1rr-2004-8/ Max Planck Institute for Gravitational Physics, Germany (2004)

27. Leibundgut, B.: Annu. Rev. Astron. Astrophys. 39, 67 (2001)

28. Liddle, A.R.: Mon. Not. R. Astron. Soc. 351, L49 (2004)

29. Niemeyer, J.C., Schmidt, W., Klingenberg, C.: Nucl. Phys. A 758, 431 (2005)

30. Oliveira, F.J., Hartnett, J.G.: Found. Phys. Lett. 19, 519 (2006)

31. Öztaş, A.M., Smith, M.L.: Int. J. Theor. Phys. 45, 925 (2006)

32. Peebles, P.J.E.: Principles of Physical Cosmology, pp. 70-131. Princeton University Press, Princeton (1993)

33. Perlmutter, S., Aldering, G., Goldhaber, G., Knop, R.A., Nugent, P., Castro, P.G., Deustua, S., Fabbro, S., Goobar, A., Groom, D.E., Hook, I.M., Kim, A.G., Kim, M.Y., Lee, J.C., Nunes, N.J., Pain, R., Pennypacker, C.R., Quimby, R., Lidman, C., Ellis, R.S., Irwin, M., McMahon, R.G., Ruiz-Lapuente, P., Walton, N., Schaefer, B., Boyle, B.J., Filippenko, A.V., Matheson, T., Fruchter, A.S., Panagia, N., Newberg, H.J.M., Couch, W.J.: Astrophys. J. 517, 565 (1999)

34. Riess, A.G., Strolger, L.-G., Casertano, S., Ferguson, H.C., Mobasher, B., Gold, B., Challis, P.J., Filippenko, A.V., Jha, S., Li, W., Tonry, J., Foley, R., Kirshner, R.P., Dickinson, M., MacDonald, E., Eisenstein, D., Livio, M., Younger, J., Xu, C., Dahlen, T., Stern, D.: Astron. J. 116, 1009 (1998)

35. Riess, A.G., Strolger, L.-G., Tonry, J., Casertano, S., Ferguson, H.C., Mobasher, B., Challis, P., Filippenko, A.V., Jha, S., Li, W., Chornock, R., Kirshner, R.P., Leibundgut, B., Dickinson, M., Livio, M., Giavalisco, M., Steidel, C.C., Benitez, N., Tsvetanov, Z.: Astrophys. J. 607, 665 (2004)

36. Riess, A.G. et al.: astro-ph/0611572 (2007)

37. Robinson, I.A., Kibble, B.P.: Metrologia 44, 427 (2007)

38. Sandage, A., Tammann, G.A., Saha, A., Reindl, B., Macchetto, F.D., Panagia, N.: Astrophys. J. 653, 843 (2006)

39. Schwarz, G.: Ann. Stat. 6, 461 (1978)

40. Smith, M.L., Öztaş, A.M., Paul, J.: Int. J. Theor. Phys. 45, 937 (2006)

41. Smith, M.L., Öztaş, A.M., Paul, J.: Ann. Fond. Louis Broglie 32, 61 (2007)

42. Smith, M.L., Öztaş, A.M.: Adv. Stud. Theor. Phys. 2, 1 (2008)

43. Tonry, J.L., Schmidt, B.P., Barris, B., Candia, P., Clocchiatti, A., Coil, A.L., Filippenko, A.V., Garnavich, P., Hogan, C., Holland, S.T., Jha, S., Kirchner, R.P., Krisciunas, K., Leibundgut, B., Li, W., Matheson, T., Philips, M.M., Riess, A.G., Schommer, R., Smith, R.C., Sollerman, J., Spyromilio, J., Stubbs, C., Suntzeff, N.B.: Astrophys. J. 594, 1 (2003)

44. Wood-Vasey, W.H. et al.: astro-ph/0701041 (2007) 